\title{
NUEVOS REGISTROS DE PLANTAS MICOHETERÓTROFAS ACLOROFILADAS PARA LA PROVINCIA DE CORRIENTES, ARGENTINA
}

\author{
ERNESTO R. KRAUCZUK ${ }^{1}$, MANUELA E. RODRÍGUEZ ${ }^{2}$, ALICIA E. CARDOZO ${ }^{3}$ \& HÉCTOR A. KELLER ${ }^{4}$
}

\begin{abstract}
Summary: Krauczuk, E. R., M. E. Rodríguez, A. E. Cardozo \& H. A. Keller. 2013. New records of achlorophyllous mycoheterotrophic plants for the Corrientes province, Argentina. Bonplandia 22(2): 131-135.
\end{abstract}

The presence of three species of mycotrophic achlorophyllous plants is documented for the first time into the territory of Corrientes province, Argentina, on the basis of herbarium specimens. The species are illustrated by photographs and the ecological information about the places where these plants grow is given.

Key words: Burmanniaceae, Triuridaceae, Orchidaceae, Corrientes, Argentina.

\begin{abstract}
Resumen: Krauczuk, E. R., M. E. Rodríguez, A. E. Cardozo \& H. A. Keller. 2013. Nuevos registros de plantas micoheterótrofas aclorofiladas para la provincia de Corrientes, Argentina. Bonplandia 22(2): 131-135.

Se presentan nuevos registros de plantas micoheterótrofas aclorofiladas para la provincia de Corrientes, Argentina. Sobre la base de especímenes de herbario recolectados en el territorio de la provincia de Corrientes, Argentina, se documenta por primera vez la presencia de tres especies de plantas micoheterótrofas sin clorofila. Las especies son ilustradas mediante fotografías y se brinda información ecológica sobre los sitios donde las plantas fueron halladas.
\end{abstract}

Palabras clave: Burmanniaceae, Triuridaceae, Orchidaceae, Corrientes, Argentina.

\section{Introducción}

Las plantas carentes de clorofila, comúnmente llamadas "saprófitas" han sido por largo tiempo consideradas simbióticas con hongos micorrízicos. El micelio producido por el hongo se sitúa dentro de determinadas células de la epidermis y capas corticales de los órganos subterráneos de la planta, como así también por fuera de misma.
Las sustancias tomadas desde el sustrato por el hongo son convertidas por enzimas que éste produce, en sustancias que la planta puede metabolizar. Puede decirse que estas plantas parasitan al hongo y son heterótrofas porque dependen del hongo para su nutrición, absorción y metabolismo (Maas \& al., 1986).

Se conoce la existencia de seis familias con especies micoheterótrofas que habitan en la región tropical del continente americano

\footnotetext{
${ }^{1}$ Gobierno de la provincia de Misiones. Ecobiblioteca, Ministerio de Ecología y Recursos Naturales Renovables. San Lorenzo 1538, 3300 Posadas, Misiones. ernestokr@yahoo.com.ar.

${ }^{2}$ Cátedra Sistemática Teórica. Facultad de Ciencias Exactas y Naturales, Universidad Nacional de Misiones. Félix de Azara 1552,3300 Posadas, Misiones. manuelarodriguez@fceqyn.unam.edu.ar

${ }^{3}$ Cátedra Sistemática Teórica. Facultad de Ciencias Exactas y Naturales, Universidad Nacional de Misiones. Félix de Azara 1552,3300 Posadas, Misiones.cardozo@fceqyn.unam.edu.ar

${ }^{4}$ Investigador del CONICET, Facultad de Ciencias Forestales, UNaM, Instituto de Botánica del Nordeste, (CONICETUNNE, Facultad de Ciencias Agrarias), C.C. 209, 3400 Corrientes, Argentina. kellerhector@hotmail.com
} 
-Neotrópico-, estas son: Burmanniaceae, Corsiaceae, Gentianaceae, Orchidaceae, Pyrolaceae y Triuridaceae (Maas \& al., 1986). En Argentina se encuentran presentes cuatro de ellas, debido a que las Gentianáceas están representadas por especies autótrofas y las Pyroliaceae no poseen representantes en nuestra flora (IBODA, 2011).

La familia Burmanniaceae está representada en nuestro país por los géneros Apteria Nutt., Burmannia L. y Gymnosiphon Blume. El género Burmannia, posee cuatro especies ( $B$. alba Mart., B. australis Malme, B. capitata (Walter ex J.F. Gmel.) Mart. y B. flava Mart.), de las cuales $B$. capitata fue documentada exclusivamente para la provincia de Corrientes y B. flava, también para Misiones (Schinini, 1975).

El género monotípico Apteria con su especie Apteria aphylla (Nutt.) Barnhart ex Small ha sido documentado para la provincia de Misiones por Molfino (1922) no existiendo hasta el presente otra cita (Schinini, 1975).

El género Gymnosiphon está representado en la Argentina por Gymnosiphon divaricatus (Benth.) Benth. \& Hook. f., que habita en la provincia de Misiones (Keller, 2011).

Sólo existe un representante de la familia Corsiaceae en el país, Arachnitis uniflora Phil., que crece en Río Negro, Neuquén, Chubut, Santa Cruz y Tierra del Fuego (IBODA, 2011).

Dentro de la familia Orchidaceae se ha citado una sola especie micoheterótrofa nativa en la Argentina, Wullschlaegelia aphylla (Sw.) Rchb. f., mencionada sólo para la provincia de Misiones por Johnson (2001).

La familia Triuridaceae se halla representada por una sola especie en la Argentina, Peltophyllum luteum Gardn., mencionada para Misiones sobre la base de ejemplares coleccionados a principios del siglo pasado (Molfino, 1925; Maas \& al., 1986). Esta especie ha sido recientemente redescubierta para dicha provincia, donde fue hallada una población en el Dep. Gral. Manuel Belgrano, simpátrica con Gymnosiphon divaricatus (Keller, 2011).

En la presente contribución se da a conocer el hallazgo de Apteria aphylla (Burmanniaceae), Peltophyllum luteum y Wullschlaegelia aphylla en la provincia de Corrientes, lo que permite ampliar la flora micoheterotrófica de esta provincia a cinco especies. Además, los ejemplares recientemente hallados permiten ratificar la presencia de las dos primeras especies en nuestro país, cuyas últimas recolecciones en Argentina se remontan hace 93 años para el primer caso y más de un siglo para el segundo.

\section{Resultados}

\section{Burmanniaceae \\ Apteria aphylla (Nutt.) Barnhart ex Small} Fig. 1B

Fl. S.E. U.S. ed. 1. 309. 1903.

Sinonimia e iconografía en Maas \& al., 1986. Fl. Neotrop. Monograph 42: 133-136. Fig. 60. 1986.

Material examinado: ARGENTINA. Corrientes. Ituzaingó, $27^{\circ} 34^{\prime} 56,7^{\prime}$ S $-56^{\circ} 31^{\prime}$ 49,9" W, 02-III-2013, fl, Keller \& Krauczuk 11318 (CTES); Rincón Ombú, 21-I-2006, fl, Krauczuk, Cardozo \& Rodríguez 4 (CTES, SI); 21-I-2006, fl, Krauczuk, Cardozo \& Rodríguez 10 (CTES).

Distribución y fenología: Se distribuye desde el sur de Estados Unidos, norte de Perú, Bolivia, Paraguay, sur de Brasil y nordeste argentino, en Misiones y Corrientes (Dep. Ituzaingó). Florece todo el año.

Obs. ecológicas: Crece enbosquesinundables entre las hojas y troncos caídos, entre cojines de licopodios o musgos. En sabanas se la encuentra solamente por encima de los $1800 \mathrm{~m}$ de altitud (Maas \& al., 1986). En Corrientes fue hallado en la cabecera noreste del macrosistema Iberá, a $81 \mathrm{~m}$ n.s.m., en fragmentos de selva anegada con predominancia de Calophyllum brasiliense Cambess. (Calophyllaceae) y sotobosque con abundancia de Blechnum brasiliense Desv. (Blechnaceae). Las poblaciones halladas que cuentan con mayor cantidad de ejemplares son simpátricas con las de Peltophyllum luteum y crecen principalmente entre los neumatóforos fibrosos que rodean la base de ejemplares de Miconia chamissois Naud. (Melastomataceae), un arbolito o arbusto frecuente en los bordes de estos fragmentos con dosel arbóreo. 


\section{Orchidaceae \\ Wullschlaegelia aphylla (Sw.) Rchb. f.}

Fig. 1C

\section{Bot Zeit. 131. 1863.}

Sinonimia e iconografía: Cogn. C. A. en Urban, I. ed. C.F.P. Martius \& A. W. Eichler, Fl. Bras. 3(4): 244. Lám. 57. 1895.

Material examinado: ARGENTINA. Corrientes. Ituzaingó, Establecimiento Santo Domingo, mogote A. $27^{\circ} 40^{\prime} 16,5 " \mathrm{~S}-56^{\circ} 10^{\prime} 30,6$ " W, 03-XII-2012, fl, fr, Keller \& al. 11181 (CTES). Ídem. $27^{\circ} 40^{\prime} 18,2$ " $\mathrm{S}-56^{\circ} 10^{\prime} 48^{\prime}$ 'W, 04-XII-2012, fl, fr, Keller \& al. 11196 (CTES).

Material adicional: ARGENTINA. Misiones. Gral. Manuel Belgrano. Bernardo de Irigoyen, $26^{\circ}$ 16' 05,2" S - 53 ${ }^{\circ} 39^{\prime} 23,6$ " W, 22-II-2011, fl, fr, Keller \& Franco 9749 (CTES). San Pedro. Reserva de Biósfera Yabotí, Parque Provincial Esmeralda, camino desde Estación Biológica hacia "Pozo Preto", 25-I-2006, fl, fr, Keller \& al. 3463 (CTES).

Distribución y fenología: Es una especie que se distribuye desde América Central hasta la Argentina (Johnson, 2001), donde crece en la provincia de Misiones (Dep. Iguazú, Guaraní, Gral. Manuel Belgrano, San Pedro, Cainguás y San Ignacio) y en la provincia de Corrientes en el Dep. Ituzaingó. Florece de diciembre a marzo.

Obs. ecológicas: Enraizada en detritos húmedos y bajo condiciones sombrías en diversos ambientes boscosos densos (Johnson, 2001). En la provincia de Corrientes fue hallada creciendo entre la hojarasca, en el interior de un fragmento de selva mixta no anegada, de más de 200 ha. Dicho fragmento que estaba incluido en un campo destinado a la cría de ganado vacuno, fue sometido a clausura en el año 2004.

\section{Triuridaceae}

\section{Peltophyllum luteum Gardn.}

Fig. 1D y E

Trans. Linn. Soc. London 19: 157. t. 15, f 1-3 \& 5-6. 1845

Sinonimia e iconografía en Maas \& al., 1986. Fl. Neotrop. Monograph 40: 43-44. Fig. 16. 1986.
Material examinado: ARGENTINA. Corrientes. Ituzaingó, 27 34' 57,4” S - 56 31' 47,3” W, 02-III2013, fl, fr, Keller \& Krauczuk 11327 (CTES); Ídem, Rincón Ombú, 27 24’ 54,42” S - 56 $6^{\circ} 15^{\prime}$ 29,43” W, 12-II-2006, fl, Krauczuk 43 (CTES, SI, WAG).

Material adicional: Misiones. Gral. Manuel Belgrano, Bernardo de Irigoyen, $26^{\circ} 16^{\prime} 05,2^{\prime \prime} \mathrm{S}-53^{\circ}$ 39' 23,6” W, 22-II-2011, fl, Keller \& Franco 9750 (CTES).

Distribución y fenología: Sureste de Brasil, sur del Paraguay, y en la Argentina crece en Misiones (Dep. San Pedro y Gral. Manuel Belgrano) y en Corrientes (Dep. Ituzaingó). Florece desde enero hasta junio.

Obs.: Las muestras recolectadas en Corrientes corresponden a plantas femeninas. Este material presenta el estilo inserto en posición terminal sobre el ovario y no lateral como corresponde a la descripción presentada para el género en Maas \& al. (1986: 43). No obstante los demás caracteres se ajustan a la descripción de la especie, por lo cual dichos ejemplares han sido identificados como $P$. luteum por los especialistas en la familia.

Obs. ecológicas: Presenta una distribución discontinua e irregular en el sudoeste y Brasil central, es característica y preferencial de la selva estacional del río Paraná y Alto Paraguay (Reitz, 1989); crece en selvas, a veces entre mantillos de hojas de bambú (Maas \& al., 1986). En la provincia de Misiones, Argentina crece en selvas con ejemplares emergentes de Araucaria angustifolia (Bertol.) Kuntze (Araucariaceae), abundancia de lauráceas y sotobosque con predominancia de Psychotria suturella Müll. Arg. (Rubiaceae), con manchones esparcidos de Alsophylla setosa Kaulf (Cyatheaceae) y Merostachys clausenii Munro (Poaceae). En el último sitio de hallazgo en dicha provincia (Keller, 2011), comparte su hábitat sombrío a más de 820 m s.n.m. con otras dos plantas micoheterótrofas; Gymnosiphon divaricatus y Wullschlaegelia aphylla.

En la provincia de Corrientes fue hallada en la cabecera noreste del macrosistema Iberá, a 79 m s.n.m., en fragmentos de selva anegada con predominancia de Calophyllum brasiliense (Calophyllaceae) y sotobosque con abundancia 

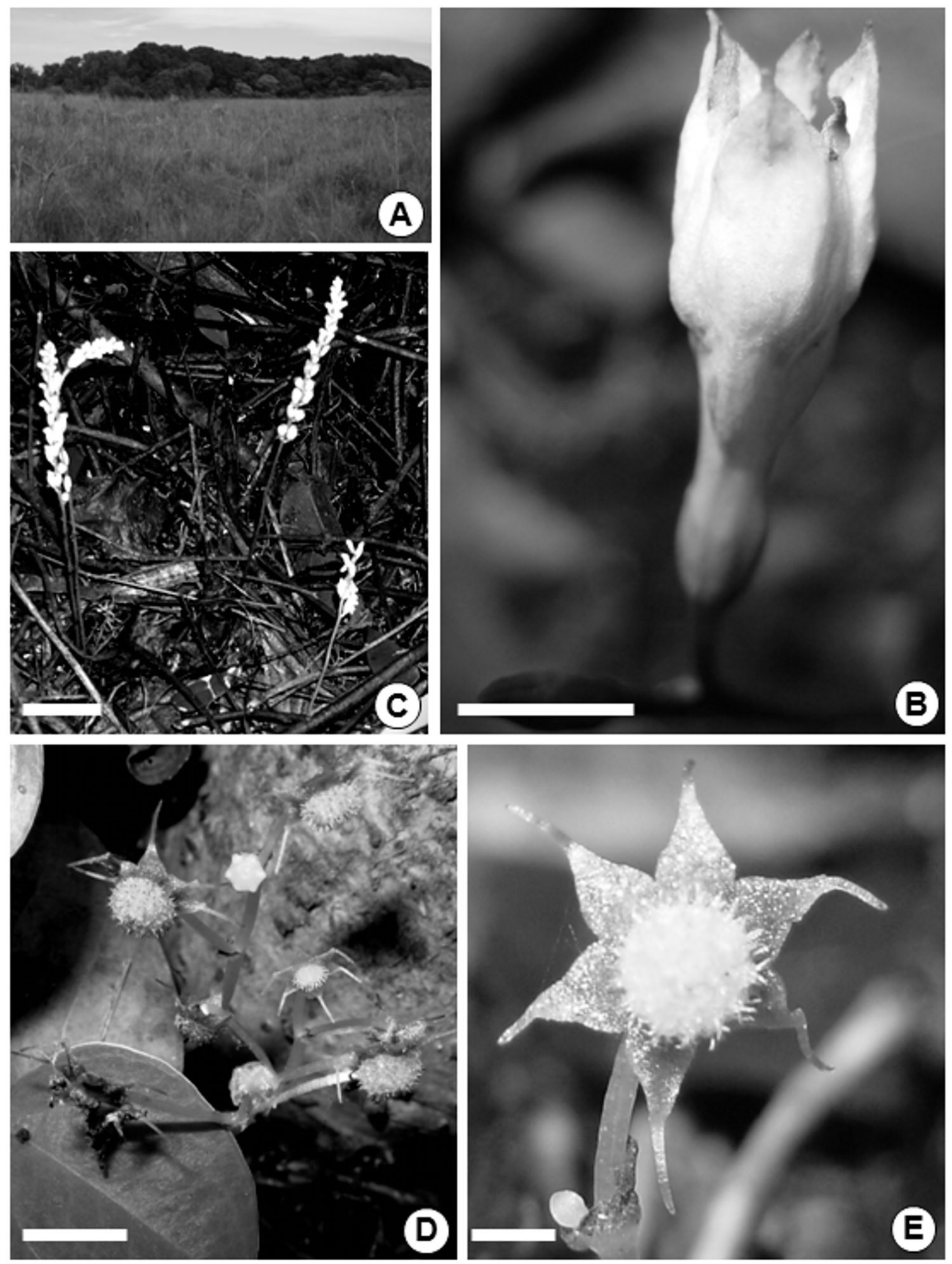

Fig. 1. A: Fragmento de selva en sector anegado, uno de los sitios de hallazgo. B: Apteria aphylla (Burmanniaceae). C: Wullschlaegelia aphylla (Orchidaceae). D y E: Peltophyllum luteum (Triuridaceae) (B: Keller \& Krauczuk 11318; C: Keller et al. 11196; D y E: Keller \& Krauczuk 11327). Escalas en cm. B: 0,3. C: 5. D: 0,5. E: 0,2. 
de Blechnum brasiliense (Blechnaceae). Las poblaciones halladas que cuentan con mayor cantidad de ejemplares son simpátricas con las de Apteria aphylla y crecen principalmente entre los neumatóforos fibrosos que rodean la base de ejemplares de Miconia chamissois Naudin, un arbolito o arbusto frecuente en los bordes de estos fragmentos con dosel arbóreo. También han sido hallados ejemplares creciendo bajo la cobertura de arbustos aislados en el estero.

\section{Agradecimientos}

A Paul. J. M. Maas y a Hiltje Maas de Wageningen University por corroborar la identificación del material de herbario. A los administradores de los establecimientos Santo Domingo y Puerto Valle, por permitirnos estudiar la flora de los predios donde fueron recolectadas las muestras. A María Mercedes Arbo y Gelina Pieszko del herbario CTES por el envío de material a los especialistas. A Luis Krause, Román Carlos Ríos y Marcelo Mauricio Franco por su colaboración durante las campañas de recolección.

\section{Bibliografía}

IBODA. 2011. Instituto de Botánica Darwinion. Base de datos. Flora del Cono Sur [online]. Disponible: http//www2.darwin.edu.ar [Acceso: 8-IV-2013].

JOHNSON, A. E. 2001. Las orquídeas del Parque Nacional Iguazú. Ed. L.O.L.A. Buenos Aires, $282 \mathrm{pp}$.

KELLER, H. A. 2011. Gymnosiphon divaricatus (Burmanniaceae), nuevo registro de una micoheterótrofa para la Argentina. Darwiniana: 49(1): 94-98.

MAAS, P. J. M., H. MAAS-VAN DE KAMER, J. VAN BENTHEM, H. C. M. SNELDERS \& T. RÜBSAMEN. 1986. Saprophytic Pro Parte. Fl. Neotrop. Monograph. 40, 41, 42: 1-189.

MOLFINO, F. J. 1922. Una Burmanniácea interesante para Misiones: "Apteria liliacina" Miers. Physis (Buenos Aires) 6: 329-330.

—. 1925. Triuridáceas, familia de fanerógamas saprófitas, nueva para la Flora Argentina. Physis (Buenos Aires) 8: 257-258.

REITZ, R. 1989. Triuridáceas en R. Reitz (Ed.): Flora Ilustrada Catarinense. Itajaí, Santa Catarina, Brasil. 12 pp.

SCHININI, A. 1975. Notas sobre Burmanniáceas argentinas. Bol. Soc. Argent. Bot. 16 (4): 349354.

Original recibido el 25 de agosto de 2013; aceptado el 7 de octubre de 2013. 
